\title{
Multiobjective Optimization Problem of Multireservoir System in Semiarid Areas
}

\author{
Z. J. Chen, ${ }^{1,2}$ Z. J. Cheng, ${ }^{1}$ and X. Q. Yan ${ }^{1}$ \\ ${ }^{1}$ Electrical Engineering College, XinJiang University, XinJiang, Urumqi 830046, China \\ ${ }^{2}$ Dapartment of Automation, Electrical Engineering College, XinJiang University, XinJiang, Urumqi 830046, China
}

Correspondence should be addressed to Z. J. Chen; chenzj1110@163.com

Received 27 June 2012; Accepted 6 September 2012

Academic Editor: Zidong Wang

Copyright (c) 2013 Z. J. Chen et al. This is an open access article distributed under the Creative Commons Attribution License, which permits unrestricted use, distribution, and reproduction in any medium, provided the original work is properly cited.

With the increasing scarcity of water resources, the growing importance of the optimization operation of the multireservoir system in water resources development, utilization, and management is increasingly evident. Some of the existing optimization methods are inadequate in applicability and effectiveness. Therefore, we need further research in how to enhance the applicability and effectiveness of the algorithm. On the basis of the research of the multireservoir system's operating parameters in the Urumqi River basin, we establish a multiobjective optimization problem (MOP) model of water resources development, which meets the requirements of water resources development. In the mathematical model, the domestic water consumption is the biggest, the production of industry and agricultural is the largest, the gross output value of industry and agricultural is the highest, and the investment of the water development is the minimum. We use the weighted variable-step shuffled frog leaping algorithm (SFLA) to resolve it, which satisfies the constraints. Through establishing the test function and performance metrics, we deduce the evolutionary algorithms, which suit for solving MOP of the scheduling, and realize the multiobjective optimization of the multireservoir system. After that, using the fuzzy theory, we convert the competitive multiobjective function into single objective problem of maximum satisfaction, which is the only solution. A feasible solution is provided to resolve the multiobjective scheduling optimization of multireservoir system in the Urumqi River basin. It is the significance of the layout of production, the regional protection of ecological environment, and the sufficient and rational use of natural resources, in Urumqi and the surrounding areas.

\section{Overview}

Xinjiang is the most north-west province in China. It is located in the inland semiarid area, which accounts for 3\% of water resources in China. The average annual surface water runoff in Xinjiang is 88.2 billion cubic meters and 5146 cubic meters per capita, which is 2.25 times the average of China [1]. The average annual groundwater exploitation is about 25.1 billion cubic meters. In addition, the glaciers reserves in Xinjiang accounted for 50 percent of China's total. Yet, Xinjiang is located in the hinterland of the Eurasian continent; the climate is dry; it is affected by seasonal factors easily. So, the spatial and temporal distribution of the water resource is extremely uneven [2].

As the capital and the center of the cultural, economic of the Xinjiang Uygur autonomous region, the population of Urumqi city is nearly 5 million. The urban water resources are mainly provided by the Urumqi River basin, which belongs to the northern slope of Tianshan inland river system. It consists of the Urumqi River, Nanshan River, Dongshan River, Toutunhe River, and so forth. Urumqi River originates in the Glacier number 1 of Tianger peak, crosses the urban area of Urumqi city, and, finally, flows into Beishawo in the southern margin of Junggar basin. Its length is $214 \mathrm{~km}$; drainage area is 5803 square kilometers; average annual runoff is 2.37 million cubic meters. Along the Urumqi River basin, there are more than 30 reservoirs as follows: Daxigou reservoir, Ulabo reservoir, Hongyanchi reservoir, Mengjin reservoir, and so on. It provides domestic water, farmland and green space irrigation water, industrial water, and aquaculture water for the residents in Urumqi city and the surrounding area. Meanwhile, it also plays an important role in flood control, drought resistance, and others [3]. 
In inland arid and semiarid regions, such as Urumqi, the shortage of water resources has become a major restricting factor of the city's development. With the rapid development of the city as well as the gradual deterioration of the ecological environment, severe water shortage has become the bottleneck of the constraints and limitations of social and economic development in Urumqi. In order to alleviate this problem, in the beginning of this century, "YinEJiU" project was implemented. Using hydraulic engineering, part of northern Xinjiang Irtysh water was injected into the Urumqi River. With the implementation of this project, a new water source is added into the Urumqi River basin. It alleviated the status of the serious water shortage in Urumqi to a certain extent. However, due to the limited increase of the water resources, it cannot fully meet the demand. Therefore, from the management point of view, in addition to the plan of reservoirs construction in the upstream, we can also apply the multiobjective optimization operation. In this way, we can alleviate the contradiction between the supply and the demand of the water resource at maximum and realize the optimal allocation of water resources in Urumqi river basin gradually.

Since the early 1960s, the research on the optimization operation of the multireservoir system has been carried out in China. The research developed rapidly after the 1980s and got a large amount of achievements. Dong introduced the principle and contents of the dynamic programming optimization technique and its application in the hydro power station reservoir (group) scheduling, as well as the principle of the multiobjective decision. Feng established the theoretical foundation for the multiobjective optimum scheduling of the reservoirs. He also discussed the basic principle and method of the large-scale system decomposition and coordination systematically. Wang introduced the theory and application of the large-scale system optimization. Using the fuzzy identification mode, Li achieved the level characteristic of the sample and the contribution rate of the relevant factors in the evaluation process of the evaluation, which aims at the comprehensive evaluation of the reservoir water resources carrying capacity. At the same time, he achieved assessment of water resources carrying capacity in regional reservoir [4].

In the recent decades, several researchers (Houck, Yakowitz, and Needham et al.) proposed methods applied in optimization operation of multireservoir system, such as linear programming, nonlinear programming, and dynamic planning. Although those optimization methods provide a way to resolve the problem of reservoir group scheduling, there are also some problems. First, some models need a lot to simplify; therefore, their simulation performance is poor. Second, some of the nonlinear methods are restricted in practical application because of the lack of the boundary conditions and more parameters. Moreover, those methods are hard to simulate the experience of scheduling knowledge. Consequently, the research on the optimization scheduling is more, and the practical application is less [5].

In the process of the multireservoir system operation, the regulation and scheduling consist of the conflicting and the intereffecting multitargets. In a specific field, people often encounter the matching problem which requires multiple solutions to achieve optimization at the same time. It is the multiobjective optimization problem, MOP. The validation of MOP is whether the optimization target is more than one and requires the simultaneous processing. In engineering applications, the MOP is very significant. These engineering applications are very complicated and difficult. They are also one of the main research fields. Since the early nineteen sixties, the MOP has attracted more and more researchers' attention from different fields. For this reason, it has very important research value and practical significance to solve MOP [6].

There are a large number of monographs focusing on the methods to resolve the MOP. In general, these books can be divided into two categories. One uses the classical optimization methods, and the other uses the methods based on evolutionary algorithm which simulates the biological evolution or activity behavior. Because evolutionary algorithm's constraint conditions are low and solution quality is high, they have been widely applied in resolving the complex optimization problem. At present, the popular evolutionary algorithm to resolve the MOP is as follows: the multiobjective genetic algorithm [7], the artificial ant colony algorithm [8], the multiobjective particle swarm optimization algorithm [9], and the shuffled frog leaping algorithm [10].

The shuffled frog leaping algorithm (SFLA) is a biological evolutionary algorithm based on swarm intelligence, which was proposed by Eusuff and Lansey in 2003. The SFLA has the characteristics of simple concept, fewer parameters, fast calculation speed, good global search ability, easy to implement, and so on. So at present, it is wildly applied to the fields of the network of water resources allocation issues, the function optimization, the multiproduct pipeline network optimization, the combinatorial optimization, the image processing, the multiuser detection, and so forth. Also, the method achieved good results. As a kind of optimization method, the SHLA has certain advantages in the optimization operation of the multireservoir system and has broad application prospects [11-13].

The purpose of this paper is to study the operation parameters. Based on these parameters, we set up the operation mathematical model and use the SFLA to realize the optimization allocation of the multireservoir system in Urumqi River basin [14].

\section{The Description of the MOP}

Using words, the MOP can be described as an optimization problem, which consists of $D$ decision variable parameters, $N$ objective functions, and $m+n$ constraints. The decision variables, the objective function, and the constraint condition are function relation. In noninferior solution set, decision makers can only choose a satisfactory noninferior solution as the final solution according to the specific requirements of the problem. The mathematic form of MOP can be described as follows:

$$
\begin{array}{r}
\min (y)=f(x)=\left[f_{1}(x), f_{2}(x), \ldots, f_{n}(x)\right], \\
n=1,2, \ldots, N
\end{array}
$$




$$
\begin{array}{ll}
\text { s.t. } & g_{i}(x) \leq 0, \quad i=1,2, \ldots, m \\
& h_{j}(x)=0, \quad j=1,2, \ldots, k \\
& x=\left[x_{1}, x_{2}, \ldots, x_{d}, \ldots, x_{D}\right] \\
& x_{d \text {-min }} \leq x_{d} \leq x_{d \text {-max }}, \quad d=1,2, \ldots, D,
\end{array}
$$

where $x$ is the decision-making relative to the amount of the $D$-dimensional, $y$ is the target vector, $g(x) \leq 0$ is the $i$ th inequality constraint, $h_{j}(x)=0$ is the $j$ th equality constraints, $f_{n}(x)$ is the $n$th objective function, $X$ the decision space formed by the decision vector, $g_{i}(x) \leq 0$ and $h_{j}(x)=0$ determine the feasible solution domain, and $x_{d \text {-min }}$ and $x_{d \text {-max }}$ are upper and lower searching limits of each dimension vector [15].

For optimal solution or noninferior optimal solution in the MOP, we can define the following.

Definition 1. For any $d \in[1, D]$ to meet $x_{d}^{*} \leq x_{d}$ and exits $d_{0} \in[1, D]$, there is $x_{d}^{*} \leq x_{d 0}$, then the vector $x^{*}=\left[x_{1}^{*}\right.$, $\left.x_{2}^{*}, \ldots, x_{D}^{*}\right]$ dominates the vector $x=\left[x_{1}, x_{2}, \ldots, x_{D}\right]$.

If $f\left(x^{*}\right)$ dominates $f(x)$, the following two conditions must be met:

$$
\begin{aligned}
& \forall n, \quad f_{n}\left(x^{*}\right) \leq f_{n}(x), \quad n=1,2, \ldots, N, \\
& \exists n_{0}, \quad f_{n_{0}}\left(x^{*}\right)<f_{n_{0}}(x), \quad 1 \leq n_{0} \leq N .
\end{aligned}
$$

The dominating relation of $f(x)$ is consistent with the dominating relation of $x$.

Definition 2. The Pareto optimal solution cannot be dominated by any solution in the feasible solution set. If $x^{*}$ is a point in the searching space, $x^{*}$ is the noninferior optimal solution. If and only if exit $x$ (in the feasible domain of the searching space), it makes the establishment of $\left(x^{*}\right)<f(x)$, $n=1,2 \ldots, N$.

Definition 3. Given a MOP of $f(x)$, if and only if for any $x$ (in the searching space), there is $f_{n}(x)<f_{n}\left(x^{*}\right) ; f\left(x^{*}\right)$ is the global optimal solution.

Definition 4. The solution set consisted of all the noninferior optimal solutions is called Pareto optimal set of the MOP, also called acceptable solution set or efficient solution set.

\section{The Description of the Shuffled Frog Leaping Algorithm (SFLA)}

The SFLA is a kind of cooperative evolutionary algorithm enlightened by natural biological mimics, which simulates the information exchange classified by subgroup in the process of the frog group looking for food. First, we generate a group of initial solution (population) from solution space randomly. Then, we divide the whole population into more than one subpopulation, in which the frogs execute subpopulation internal search according to a certain strategy. After the end of the number of searches in defined subpopulation, mix all the frogs, sort and divide the subpopulation again, and exchange information between each subpopulation globally. The sub-population internal search and the global information exchange continue alternating, until they satisfy the convergence condition or reach the maximum number of evolution. The following is mathematical model of the SHLA.

3.1. Dividing Subpopulation. Suppose that the number of frogs in the population (candidate solution) is $N$, the number of subpopulation is $k$, the number of candidate solutions is $n . X_{j}=\left(x_{j 1}, x_{j 2}, \ldots, x_{j D}\right)$ represents the $j$ th candidate solution, $j(0 \leq j \leq N)$, and $D$ represents the dimensions of the candidate solution. For the initial population $S=$ $\left(X_{1}, X_{2}, \ldots, X_{N}\right)$ generated randomly, after sorted according to the fitness $f(x)$ in descending, the first candidate solution is assigned to the first subpopulation, the second candidate solution is assigned to the second subpopulation,..., the $k$ th candidate solution is assigned to the $k$ th subpopulation, the $(k+1)$ th candidate solution is assigned to the first subpopulation, and the $(k+2)$ th candidate solution is assigned to the second subpopulation, repeat until $N$ candidate solutions are distributed.

3.2. Internal Search in Subpopulation. Suppose that $X_{b}$ is the candidate solution, in which fitness is best in the subpopulation, $X_{w}$ is the candidate solution, in which fitness is worst in the subpopulation, and $X_{m}$ is the candidate solution, in which fitness is best in the whole population. For every subpopulation, we do internal search. It is to update $X_{w}$ of the subpopulation. The search strategy is as follows:

$$
X^{\prime}=X_{w}+R \times\left(X_{b}-X_{w}\right),
$$

where $X^{\prime}$ is the new solution produced by (3) and $R$ is a random number between 0 and 1 . If $X^{\prime}$ is superior to $X_{w}$, then $X_{w}=X^{\prime}$. Otherwise, $X_{b}$ is replaced by $X_{m}$ in (3) and repeats the search strategy. If $X^{\prime}$ is not always superior to $X_{w}$, a random candidate solution is generated to replace $X_{w}$. We repeat the above steps until the number of searches is greater than the largest number of the largest subpopulation internal search.

3.3. Global Information Exchange. When all the internal updates of subpopulation are completed, we do the subpopulation dividing and internal search again, until it meet the termination conditions (converges to the optimal solution or reaches the maximum number of evolving generation).

\section{Reservoir Group Optimization Dispatching of Urumqi River Basin}

In the process of multireservoir system optimization operation of Urumqi River basin, we should consider the following five aspects of the requirements: first, to satisfy the demand for residential water; second, to satisfy the water demand of the industrial and the agricultural production; third, to satisfy the water demand of the ecological environment; fourth, to complete the industrial and the agricultural planning output 
target; last, the investment of water resources development is minimum, under the premise to satisfy the demand of water. We consider these five aspects, which are mutual compensation, to achieve the most satisfactory results, under the condition that satisfies the system performance [16].

4.1. The Objective Functions of Multireservoir System Optimization Operation. With the above, we take the following as the goal function: people living water consumption is the largest, the industrial and the agricultural production water consumption is the largest, ecological environment water consumption is the largest, the gross output value of industry and agriculture is the highest, and the investment of water resources development is minimum [17].

According to the MOP, the objective function, the decision variables, and the constraints of the reservoir group optimization dispatching can be described as follows:

$$
\begin{array}{ll}
\min / \max & f_{m}\left(x_{1}, x_{2}, \ldots, x_{n}\right), \quad m=1,2, \ldots, M ; \\
\text { s.t. } & \text { (1) } g_{k}\left(x_{1}, x_{2}, \ldots, x_{n}\right) \geq 0, \quad k=1,2, \ldots, M ; \\
& \text { (2) } h_{l}\left(x_{1}, x_{2}, \ldots, x_{n}\right)=0, \quad l=1,2, \ldots, L ; \\
& \text { (3) } x_{i}^{(L)} \leq x_{i} \leq x_{i}^{(U)}, \quad i=1,2, \ldots, n,
\end{array}
$$

where $X \subset R^{n}$ is the decision space, $x=\left(x_{1}, x_{2}, \ldots, x_{n}\right) \in X$, and $x_{i}^{(L)}$ and $x_{i}^{(H)}$ are upper and lower bounds of variables, respectively. Further, we can define collection $\Omega$ as the feasible domain of (4):

$$
\begin{gathered}
\Omega=\left\{x \mid g_{k}\left(x_{1}, \ldots, x_{n}\right) \geq 0, h_{k}\left(x_{1}, \ldots, x_{n}\right)=0,\right. \\
\left.x_{i}^{(L)} \leq x_{i} \leq x_{i}^{(U)}\right\}, \quad \Omega \subset X .
\end{gathered}
$$

Among the objective functions of multireservoir system optimization operation, the investment of water resources development is the minimizing objective function, and the others are the maximum objective functions. In order to maintain unity, by taking the negative of the water resources investment, we convert all the problems into the maximum objective functions MOP. The objective functions can be described as follows:

$$
\begin{aligned}
& \max f_{1}=\sum_{t=1}^{T} \sum_{i}^{I} a 1_{i} P_{G i(t)}^{2}+\beta 1_{i} P_{G i(t)}+\gamma 1_{i}, \\
& \max f_{2}=\sum_{t=1}^{T} \sum_{j}^{J} a 2_{j} P_{S j(t)}^{2}+\beta 2_{j} P_{S j(t)}+\gamma 2_{j}, \\
& \max f_{3}=\sum_{t=1}^{T} \sum_{k}^{K} a 3_{k} P_{W k(t)}^{2}+\beta 3_{k} P_{W k(t)}+\gamma 3_{k}, \\
& \max f_{4}=\sum_{t=1}^{T} \sum_{l}^{L} a 4_{l} P_{H l(t)}^{2}+\beta 4_{l} P_{H l(t)}+\gamma 4_{l}, \\
& \max f_{5}=\sum_{t=1}^{T} \sum_{m}^{M} a 5_{m} P_{U m(t)}^{2}+\beta 5_{m} P_{U m(t)}+\gamma 5_{m} .
\end{aligned}
$$

For (6), $I, J, K, L$, and $M$ denote the effects of five optimal goal influence factors, respectively. $P_{G i(t)}$ is the water consumption of the $i$ th living water user in the $t$ th time interval; $P_{S i(t)}$ is the water consumption of the $j$ th industry and agriculture water user in the $t$ th time interval; $P_{W k(t)}$ is the water consumption of the $k$ th ecological water user in the $t$ th time interval; $P_{H l(t)}$ is the output value of the $l$ th production unit in the $t$ th time interval; $P_{U m(t)}$ is the investment amount of the $m$ th water resources development unit in the $t$ th time interval. $a n_{*}, \beta n_{*}, \gamma n_{*}\left(n=1,2,3,4,5 ;{ }^{*}=i, j, k, l, m\right)$ is the influence coefficient of each factor; $T$ is the scheduling cycle.

4.2. The Constraint Condition of Multireservoir System Optimization Operation. In dealing with the constraint condition, according to the objective function, we make multireservoir system operation satisfaction as constraint condition. In view of reservoir group optimal model, the constraint conditions are as follows.

The water consumption demand of people living is mainly affected by the climate change and daily change. So, the living water consumption change and its rate of change are always in a certain range. As one of the important indexes which influence the reservoir group operation, under the premise of the whole objective optimization and reducing the consumption of water, as far as possible, the constraint conditions of the living water consumption can be described as follows:

$$
\begin{gathered}
P_{G \min }<\sum P_{G}<P_{G \max }, \\
\Delta \sum P_{G}<\Delta P_{G \max },
\end{gathered}
$$

where $\sum P_{G}$ is the living water consumption of the reservoir group system in unit interval (usually a day). $\Delta \sum P_{G}$ is the variation of the living water consumption of the reservoir group system in unit interval. $P_{G \min }$ and $P_{G \max }$ are the minimum and the maximum living water consumption, respectively. $\Delta P_{G \max }$ is the largest living water quantity variation in unit interval [18].

The industrial and agricultural water consumption is directly related to the production situation. In addition to this, the agricultural water consumption is also affected by the weather, the rainfall, the plant growth period, and other factors. The industrial water consumption is also affected by the environment temperature. As another important indicator of the reservoir group operation satisfaction, the constraint conditions of the industrial and agricultural water consumption can be described as follows:

$$
\begin{gathered}
P_{S \min }<\sum P_{S}<P_{S \max }, \\
\Delta \sum P_{S}<\Delta P_{S \text { max }},
\end{gathered}
$$

where $\sum P_{S}$ is the industrial and the agricultural water consumption of the reservoir group system in unit interval (usually a day). $\Delta \sum P_{S}$ is the variation of the industrial and the agricultural water consumption of the reservoir group system in unit interval. $P_{S \text { min }}$ and $P_{S \max }$ are the minimum and the maximum industrial and agricultural water consumption, 
respectively. $\Delta P_{S \max }$ is the largest industrial and agricultural water quantity variation in unit interval [19].

The ecological environmental water consumption is the other important satisfaction index of the reservoir group operation. The constraint conditions of the ecological environmental water consumption can be described as follows:

$$
\begin{gathered}
P_{W \min }<\sum P_{W}<P_{W \max }, \\
\Delta \sum P_{W}<\Delta P_{W \text { max }},
\end{gathered}
$$

where $\sum P_{W}$ is the ecological environmental water consumption of the reservoir group system within the unit interval (usually a day). $\Delta \sum P_{W}$ is the variation of the ecological environmental water consumption of the reservoir group system within the unit interval. $P_{W \min }$ and $P_{W \max }$ are the minimum and the maximum industrial and agricultural water consumption, respectively. $\Delta P_{W \max }$ is the largest ecological environmental water quantity variation within the unit interval. The ecological environmental water consumption is influenced by the climate, environment, and external factors, and it relates to the living water consumption, the industrial, and agricultural water consumption.

The gross output value of industry and agriculture is one of the most important satisfaction indexes of the reservoir group operation. We can describe it with the social gross output. So its constraint conditions can be described as follows:

$$
\sum P_{H} \geq P_{H \text { min }}
$$

where $\sum P_{H}$ is the gross output value of industry and agriculture in a unit of time (usually a month), and $\Delta P_{H \text { min }}$ is the minimum gross output value in unit interval.

It is the necessary conditions for the investment of the water resources development to ensure the normal operation of the reservoir group. Under the general condition, the minimum investment maximizes the benefits. So, the constraint conditions of the investment of the water resources development can be described as follows:

$$
\begin{gathered}
\sum P_{U} \leq P_{U \text { max }}, \\
\frac{\sum P_{U}}{\operatorname{Count}\left(P_{U}\right)} \geq \bar{P}_{U \text { min }},
\end{gathered}
$$

where $\sum P_{U}$ is the investment of the water resources development, $P_{U \text { max }}$ is the maximum investment of the water resources development, $\operatorname{Count}\left(P_{U}\right)$ is the water resources investment project number, and $\bar{P}_{U \text { min }}$ is the minimum social product of the individual water resources of the investment project.

4.3. Solving the MOP of the Reservoir Group Optimization Dispatching Using SFLA. For evaluation of each frog (the solution), the following fitness function can be used:

$$
f(x)=\frac{1}{J+\alpha},
$$

where $J$ is the maximum value of the solutions, which is maximum satisfaction; $\alpha(0<\alpha<0.1)$ is a constant. Obviously,

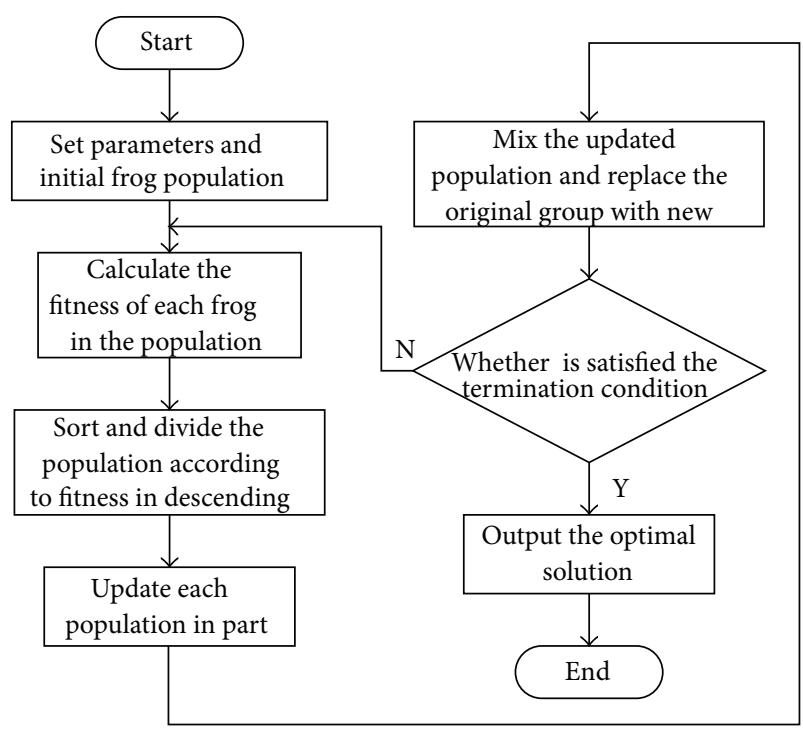

FIGURE 1: The flow chart of SFLA.

the smaller the value of the objective function, the greater the fitness of frog.

We generate $L$ frogs (the solution of the problem) as the initial population; the update strategies of each population local depth search are as follows:

$$
S=\left\{\begin{array}{r}
U(q)=P_{W}+S \\
\min \left\{\operatorname{int}\left[\operatorname{rand}\left(P_{B}-P_{W}\right)\right], S_{\max }\right\} \\
\text { Positive direction } \\
\max \left\{\operatorname{int}\left[\operatorname{rand}\left(P_{B}-P_{W}\right)\right],-S_{\max }\right\} \\
\text { Negative direction, }
\end{array}\right.
$$

where $U(q)$ is the present moment solution, $P_{W}$ is the last moment solution, $S$ is the moving distance on the components, rand is a random number between 0 and 1 , and $P_{B}$ and $P_{W}$ are the optimal and worst solution of the fitness. The steps of the SFLA are as follows.

Step 1. Set parameters and initial frog population.

Step 2. Calculate the fitness of each frog in the population.

Step 3. Sort and divide the population according to fitness in descending.

Step 4. Update each population in part.

Step 5. Mix the updated population and replace the original group with new.

Step 6. If it meets the termination condition, output the optimal solution and finish operation; if not, go Step 2 .

The program flow chart is shown in Figure 1.

4.4. The Improvement of the SFLA: The Weighted Variable Step SFLA. Through studying the basic principle of the SFLA and 


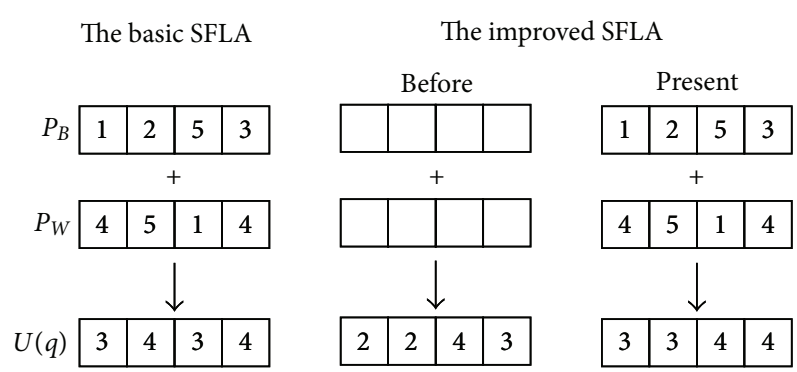

FIgURE 2: The effect of the improved SFLA.

in order to solve the engineering problems using the SFLA, it is necessary to improve algorithm efficiency and search breadth. The method using the weighted variable step-size search is adopted. We call this method the weighted variable step SFLA.

In the process of the basic SFLA, for each decision variable is the same value in rand. So it will limit the random of the decision variables to the optimal direction. This problem will be avoided, if each random decision variable generates different rand values. The step formula can be improved as follows:

$$
S^{(i)}=\left\{\begin{array}{r}
\min \left\{\operatorname{int}\left[\operatorname{rand}^{(i)}\left(P_{B}^{(i)}-P_{W}^{(i)}\right)\right], S_{\max }\right\} \\
\text { Positive direction } \\
\max \left\{\operatorname{int}\left[\operatorname{rand}^{(i)}\left(P_{B}^{(i)}-P_{W}^{(i)}\right)\right],-S_{\max }\right\} \\
\text { Negative direction. }
\end{array}\right.
$$

At the same time, in order to keep solution with better fitness and eliminate the solution with bad fitness, to each decision variable, rand is multiplied by a variable weight coefficient which relates to the fitness. In order to reflect the global search, steps do not only relate to present value, but also relate to before value. The step formula can be improved as follows:

$$
\begin{aligned}
& S^{(i)} \\
& \min \left\{\operatorname{int}\left[\alpha_{f(x)} \operatorname{rand}^{(i)}\left(P_{B}^{(i)}-P_{W}^{(i)}\right)+\beta_{f(x)} S^{(i-1)}\right], S_{\max }\right\} \\
& \text { Positive direction } \\
& \max \left\{\operatorname{int}\left[\alpha_{f(x)} \operatorname{rand}^{(i)}\left(P_{B}^{(i)}-P_{W}^{(i)}\right)+\beta_{f(x)} S^{(i-1)}\right],-S_{\max }\right\} \\
& \text { Negative direction. }
\end{aligned}
$$

$\alpha_{f(x)}+\beta_{f(x)}=1\left(0<\alpha_{f(x)}, \beta_{f(x)}<1\right)$.

Figure 2 is a decision effect using the improved SFLA, compared with the basic SFLA [20].

In the process of local search, we can improve the search method; make improvements in the posterior half of the frog in the population, which is called $1 / 2$ search method. Using this kind of method, the update is more efficient; the transformation can be ensured towards the high fitness direction; the search range can be widened.

The optimization process flow of improved SFLA is shown in Figure 3.

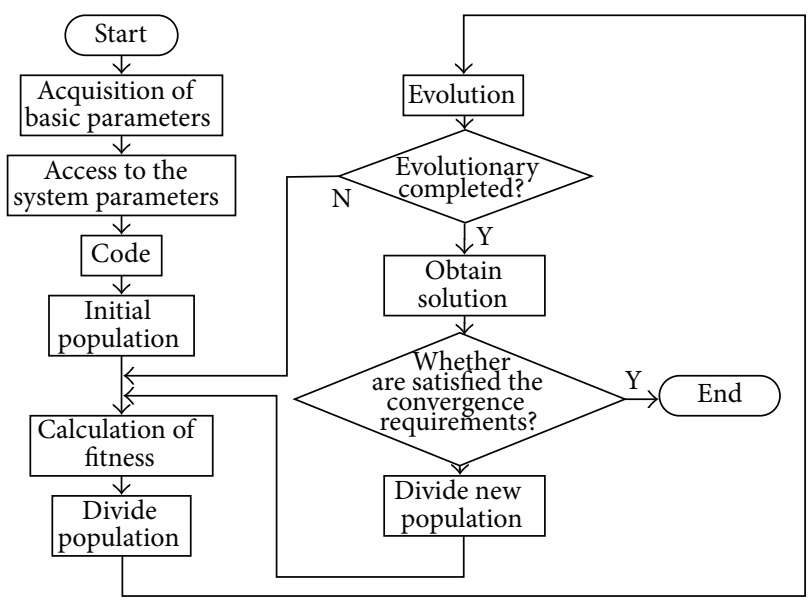

FIgURE 3: The flow chart of improved SFLA.

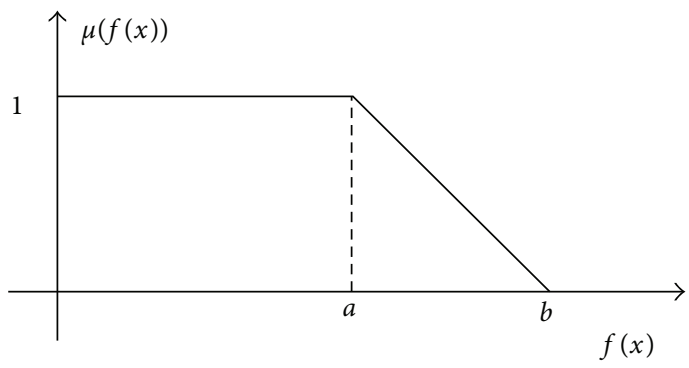

FIGURE 4: The objective membership function.

4.5. The Greatest Satisfaction Transformation of the Reservoir Group MOP. There are five competing goals of the function in the reservoir group optimization dispatching MOP. We can use the fuzzy set theory to translate them into single-objective problem, using the descending half-line as their membership function [21, 22], shown in Figure 4. follows:

The objective membership function can be described as

$$
\mu(f(x))= \begin{cases}1, & f(x)<a, \\ \frac{b-f(x)}{b-a}, & a \leq f(x) \leq b, \\ 0, & f(x)>a,\end{cases}
$$

where " $a$ " is the optimization target of the single-objective function, " $a+b$ " is the maximum acceptable values, and $\lambda$ is the minimum membership variables in all the membership function, called the degree of satisfaction;

$$
\lambda=\min \left\{\mu\left(f_{1}(x), f_{2}(x), f_{3}(x)\right)\right\} .
$$

According to the principle of maximum degree of membership, we can translate the MOP into single-objective problem with satisfaction maximization [23].

\section{The Conclusion}

For competitive multiobjective function of multireservoir system optimization operation in the inland river basin of 
semiarid areas, using fuzzy set theory, we convert the MOP of the multireservoir system into single-objective problem of maximum satisfaction. The only solution of fuzzy reasoning makes each objective function achieve optimization [24]. Using this method, we simplify the water resources optimization scheduling problem. It is advantageous for the MOP of the inland river basin of arid areas to accelerate the engineering process [25].

For the requirements of the hydrology resource and the reservoir scheduling optimization analysis, there are more and more new theories and new technologies applied to the field of water resources. In the example of Urumqi River basin, we resolved the MOP of multireservoir system optimization operation in the semiarid areas by the SFLA. The research can promote the optimal scheduling and scientific management of water resources in the semiarid inland areas [26]. With the development of the city, the problem of rational management is increasingly important. It is of great significance for the development of the cities to resolve the MOP of the water resource optimal scheduling.

In the process of research on the optimization operation of the multireservoir system, besides the appropriate optimization algorithm, the processing of the input information is essential. The input information of the system is random in essence. So the system can be considered as a nonlinear stochastic system. At present, the latest research of these issues focuses on the $H \infty$ filtering $[27,28]$ and the distributed filtering [29]. The issue will be discussed in the late papers.

\section{Acknowledgments}

This work was financially supported by Innovation Fund for Small Technology-Based Firms 11C26216506453. Also, this work was financially supported by Key Projects of the Department of Education of Xinjiang Uygur Autonomous Region XJEDU2010I07.

\section{References}

[1] B. Ye, D. Yang, K. Jiao et al., "The Urumqi River source Glacier No. 1, Tianshan, China: changes over the past 45 years," Geophysical Research Letters, vol. 32, no. 21, pp. 1-4, 2005.

[2] Z. Li, W. Wang, M. Zhang, F. Wang, and H. Li, "Observed changes in streamflow at the headwaters of the Urumqi River, eastern Tianshan, central Asia," Hydrological Processes, vol. 24, no. 2, pp. 217-224, 2010.

[3] L. H. Li, M. J. de la Paix, X. Chen et al., "Study on productivity of epilithic algae in Urumqi River Basin in Northwest China," African Journal of Microbiology Research, vol. 5, no. 14, pp. 18881895, 2011.

[4] X. Zheng, H. Guo, and B. Zhang, "Study on application of the SD-MOP mix model to urban water resource planning of Qinghuangdao city," Advances in Water Science, vol. 13, no. 3, pp. 351-357, 2002.

[5] V. E. Efimov, "Problem of water resources in the Middle East," Gigiena i Sanitariia, no. 1, pp. 21-23, 1994.

[6] C. Drake, "Water resource conflicts in the Middle East," Journal of Geography, vol. 96, no. 1, pp. 4-12, 1997.
[7] H. Ishibashi, H. E. Aguirre, K. Tanaka, and T. Sugimura, "Multiobjective optimization with improved genetic algorithm," in Proceedings of the IEEE International Conference on Systems, Man and Cybernetics, vol. 5, pp. 3852-3857, October 2000.

[8] S. Ruoying, W. Xingfen, and Z. Gang, "An ant colony optimization approach to multi-objective supply chain model," in Proceedings of the 2nd IEEE International Conference on Secure System Integration and Reliability Improvement (SSIRI '08), pp. 193-194, July 2008.

[9] E. Granger, D. Prieur, and J. F. Connolly, "Evolving ARTMAP neural networks using multi-objective particle swarm optimization," in IEEE Congress on Evolutionary Computation (CEC '10), pp. 1-8, July 2010.

[10] L. Yinghai, D. Xiaohua, L. Cuimei et al., "A modified shuffled frog leaping algorithm and its application to short-term hydrothermal scheduling," in Proceedings of the 7th International Conference on Natural Computation (ICNC '11), pp. 1909-1913, July 2011.

[11] L. Yan, X.-F. Kong, and R.-Q. Hao, "Modified shuffled frog leaping algorithm applying on logistics distribution vehicle rounting problem," in Proceedings of the 4th International Conference on the Biomedical Engineering and Informatics (BMEI '11), pp. 2277-2280, October 2011.

[12] A. Khorsandi, A. Alimardani, B. Vahidi, and S. H. Hosseinian, "Hybrid shuffled frog leaping algorithm and Nelder-Mead simplex search for optimal reactive power dispatch," IET Generation, Transmission and Distribution, vol. 5, no. 2, pp. 249-256, 2011.

[13] M. Wang and W. Di, "A modified shuffled frog leaping algorithm for the traveling salesman problem," in Proceedings of the 6th International Conference on Natural Computation (ICNC '10), pp. 3701-3705, August 2010.

[14] L. Ping, L. Qiang, and W. Chenxi, "Modified shuffled frog leaping algorithm based on new searching strategy," in Proceedings of the 7th International Conference on Natural Computation (ICNC '11), pp. 1346-1350, July 2011.

[15] L. Dayong, W. Jigan, D. Zengchuan et al., "Study on multi-objective optimization model of industrial structure based on water resources and its application," in Proceedings of the International Conference on Information Engineering and Computer Science (ICIECS '09), pp. 1-5, December 2009.

[16] H. J. Peng, M. H. Zhou, and H. H. Zhang, "Research on multiobjective collaborative optimization model and algorithm of supply chain system based on complex needs," in Proceedings of the WRI World Congress on Software Engineering (WCSE '09), pp. 424-428, May 2009.

[17] R. Bos, "Water resources development policies, environmental management and human health," Parasitology Today, vol. 6, no. 6, pp. 173-174, 1990.

[18] Z. Sanyou, C. Shizhong, Z. Jiang et al., "Dynamic constrained multi-objective model for solving constrained optimization problem," in Proceedings of the IEEE Congress on Evolutionary Computation (CEC '11), pp. 2041-2046, June 2011.

[19] G. Yinan, C. Jian, and M. Xiaoping, "Coking optimization control model based on hierarchical multi-objective evolutionary algorithm," in Proceedings of the 6th World Congress on Intelligent Control and Automation (WCICA '06), pp. 6544-6548, June 2006.

[20] M.-R. Chen, X. Li, and N. Wang, "An improved shuffled frog-leaping algorithm for job-shop scheduling problem," in Proceedings of the 2nd International Conference on Innovations 
in Bio-Inspired Computing and Applications (IBICA '11), pp. 203206, December 2011.

[21] S. Bandyopadhyay, "Multiobjective simulated annealing for fuzzy clustering with stability and validity," IEEE Transactions on Systems, Man and Cybernetics Part C, vol. 45, no. 5, pp. 682691, 2011.

[22] O. Guenounou, A. Belmehdi, and B. Dahhou, "Multi-objective optimization of TSK fuzzy models," in Proceedings of the 5th International Multi-Conference on Systems, Signals and Devices (SSD ’08), pp. 1-6, July 2008.

[23] C. L. Chen and C. P. Weng, "A multi-objective problem based on fuzzy inference with application to parametric design of an electrophotographic system," Expert Systems with Applications, vol. 38, no. 7, pp. 8673-8683, 2011.

[24] L. Zhangjun and L. Yaolong, "A new multi-objective optimization model based on fuzzy probability theory," in Proceedings of the 7th International Conference on Fuzzy Systems and Knowledge Discovery (FSKD '10), pp. 54-56, August 2010.

[25] S. Jain and K. Lachhwani, "Fuzzy programming approach to solve multi objective linear fractional program with homogeneous constraints," Proceedings of the National Academy of Sciences of India A, vol. 81, pp. 29-38, 2011.

[26] J. Zhang, H. Lu, and L. Fan, "The multi-objective optimization algorithm to a simple model of urban transit routing problem," in Proceedings of the 6th International Conference on Natural Computation (ICNC '10), pp. 2812-2815, August 2010.

[27] Z. D. Wang, B. Shen, and X. H. Liu, "H-infinity filtering with randomly occurring sensor saturations and missing measurements," Automatica, vol. 48, no. 3, pp. 556-562, 2012.

[28] W. Zidong, S. Bo, and S. Huisheng, "Quantized H-infinity control for nonlinear stochastic time-delay systems with missing measurements," IEEE Transactions on Automatic Control, vol. 57, no. 6, pp. 1431-1444, 2012.

[29] D. Hongli, W. Zidong, and G. Huijun, "Distributed filtering for a class of time-varying systems over sensor networks with quantization errors and successive packet dropouts," IEEE Transactions on Signal Processing, vol. 60, no. 6, pp. 3164-3173. 


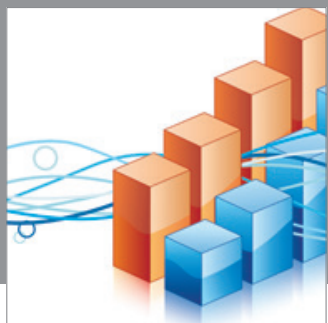

Advances in

Operations Research

mansans

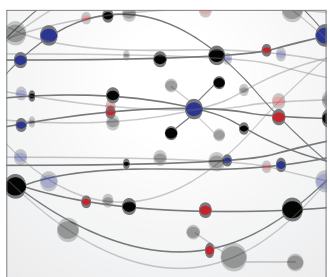

The Scientific World Journal
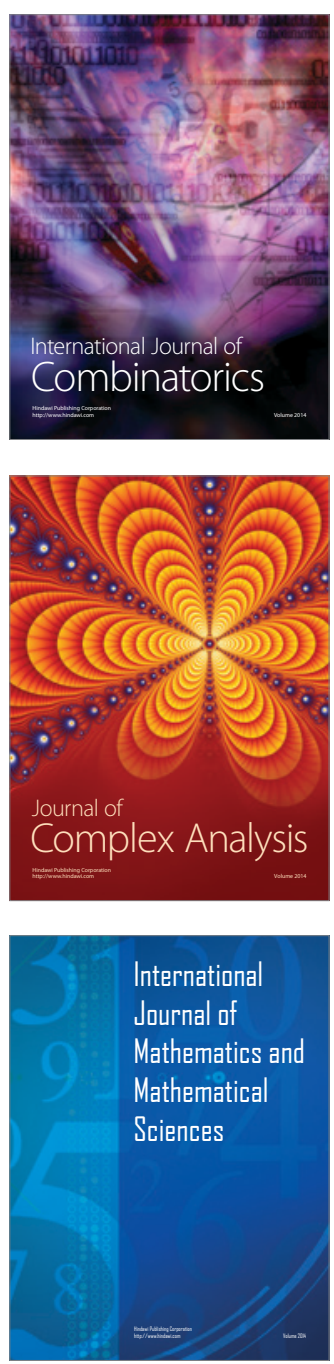
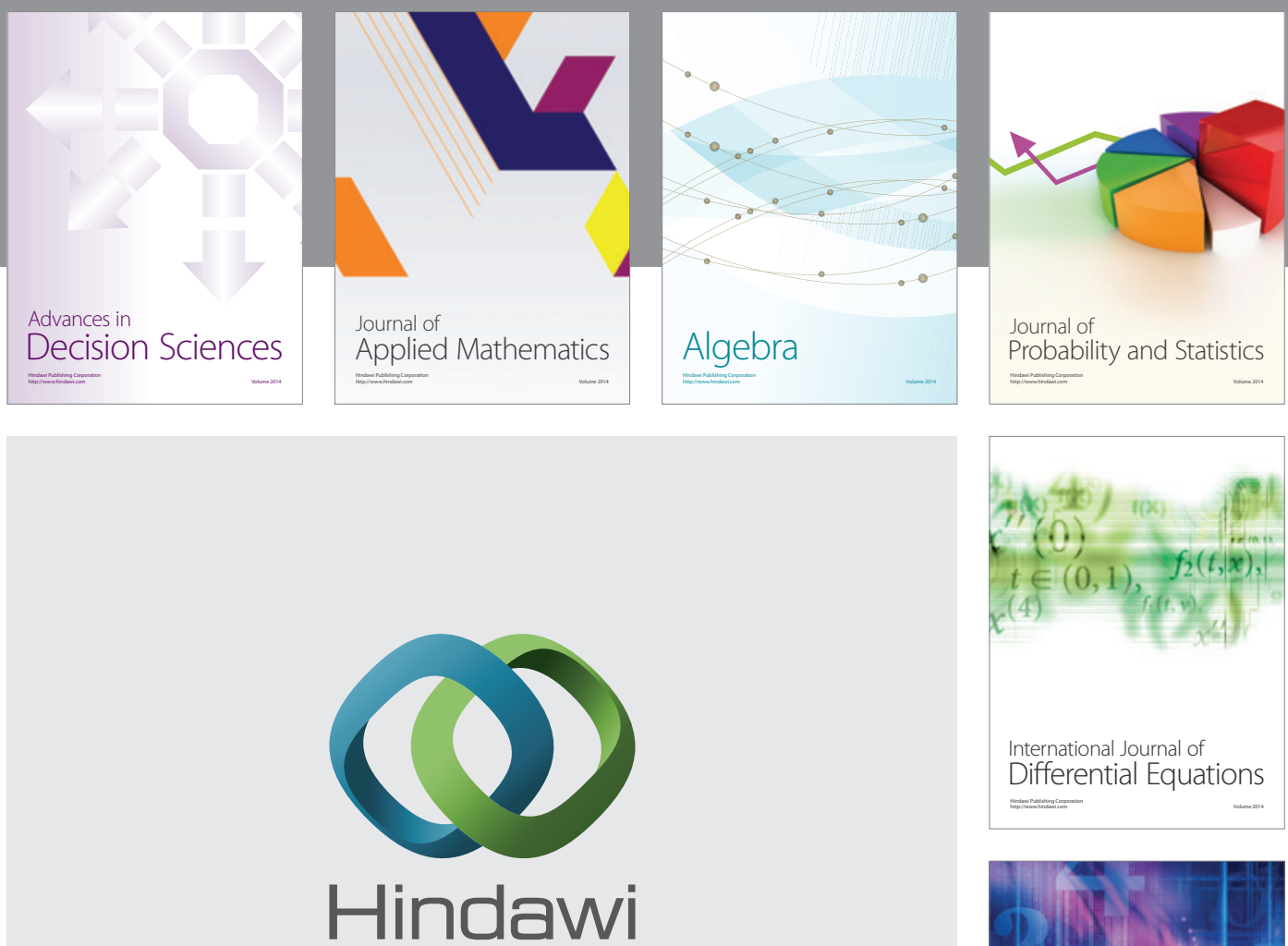

Submit your manuscripts at http://www.hindawi.com
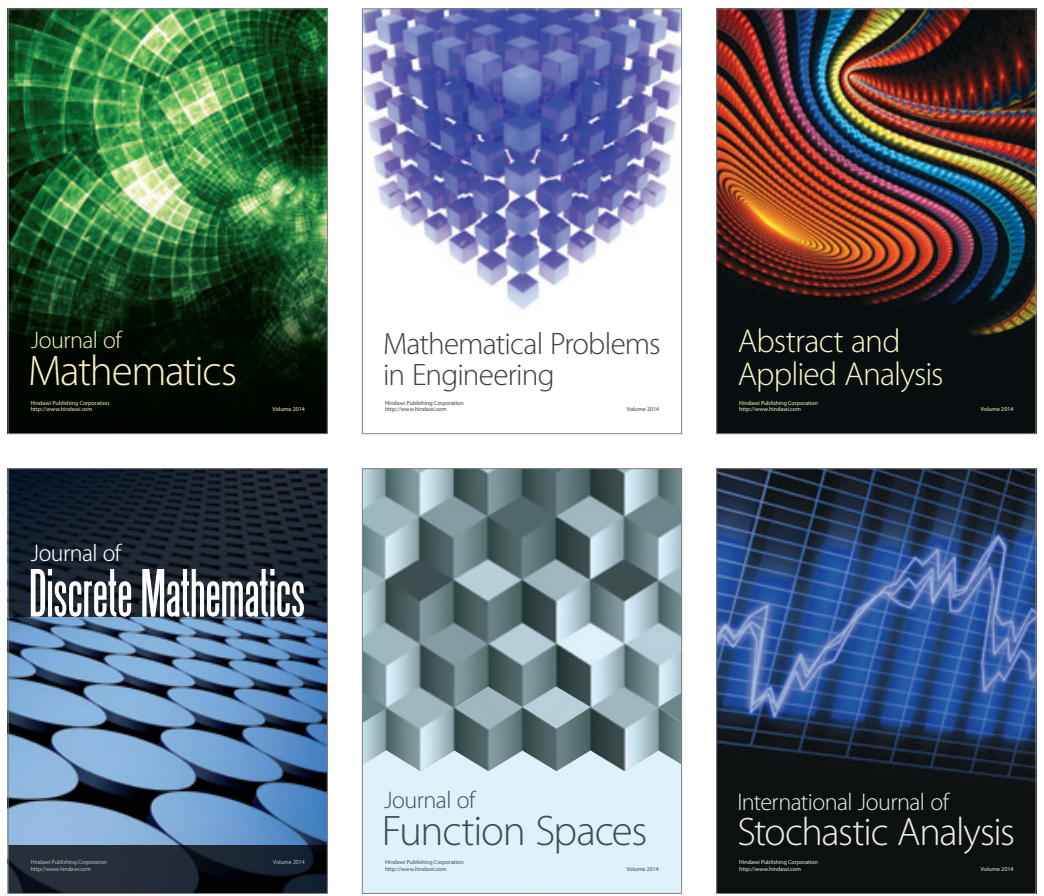

Journal of

Function Spaces

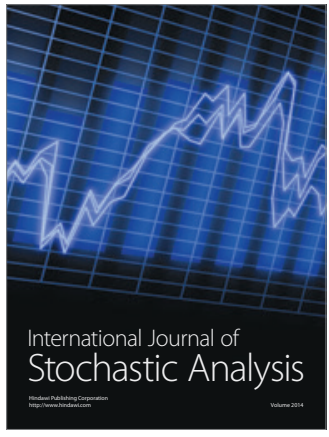

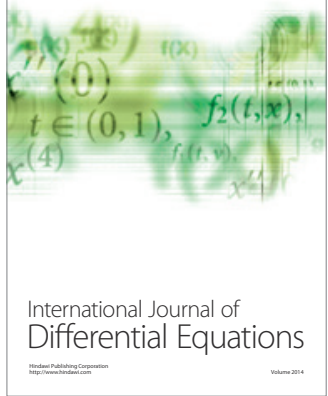
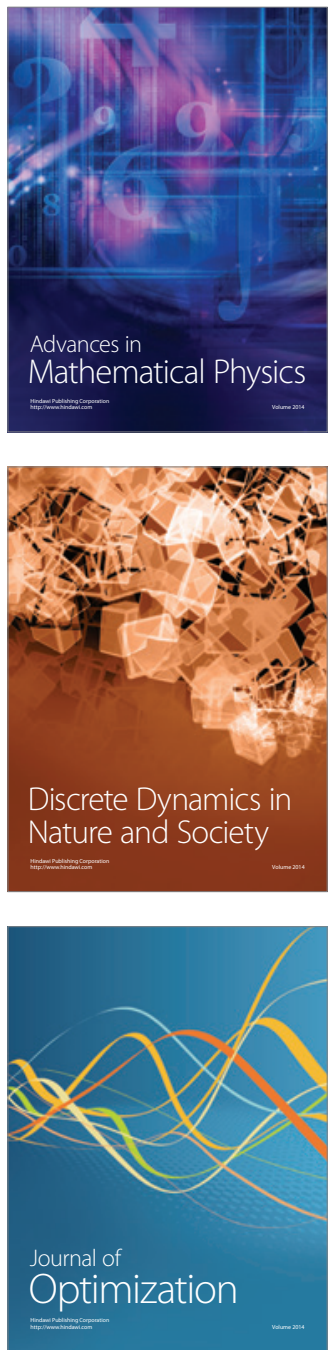